\title{
Instability of a Pseudo-Relativistic Model of Matter with Self-Generated Magnetic Field
}

\author{
Marcel Griesemer*and Christian Tix \\ Mathematik, Universität Regensburg \\ D-93040 Regensburg
}

\begin{abstract}
For a pseudo-relativistic model of matter, based on the no-pair Hamiltonian, we prove that the inclusion of the interaction with the self-generated magnetic field leads to instability for all positive values of the fine structure constant. This is true no matter whether this interaction is accounted for by the Breit potential, by an external magnetic field which is chosen to minimize the energy, or by the quantized radiation field.
\end{abstract}

\section{Introduction}

The stability of matter problem concerns the question whether the minimal energy of a system of particles is bounded from below (stability of the first kind), and whether it is bounded from below by a constant times the number of particles (stability of the second kind). Stability of the second kind for non-relativistic quantum-mechanical electrons and nuclei was first proved in 1967 by Dyson and Lenard 11, 2]. Since the new proofs of Lieb and Thirring, and Federbush in 1975 stability of matter is a subject of ongoing interest dealing with more and more realistic models of matter such as systems with a classical or quantized magnetic field included or with relativistic electrons (see [3] and the references therein). Stability with relativistic electrons is more subtle because of the uniform $1 /$ length scaling behavior of the energy, which holds for massless particles (high particle-energy limit). Then the minimal energy is either non-negative or equal $-\infty$, so that stability of the second kind becomes equivalent to the statement that stability of the first kind holds for any given number of particles. We simply call this stability henceforth.

This paper is about a pseudo-relativistic model of matter which is stable, but which becomes unstable when the electrons are allowed to interact with the self-generated magnetic field. The self-generated magnetic field may be described using either an effective potential (the Breit-potential), an external magnetic field over which the energy is minimized, or the quantized radiation field. In all these cases we find instability for all positive values of the fine-structure constant. In contrast to most other models, where the collapse of the system, if it occurs, is due to the attraction of electrons and nuclei [4, 5, 6, 7] (there would be no collapse without this interaction), the instability here is due to the attraction of parallel currents.

The model we study is based on a pseudo-relativistic Hamiltonian sometimes called no-pair or Brown-Ravenhall Hamiltonian describing $N$ relativistic electrons and $K$ fixed

${ }^{*}$ Present address: Department of Mathematics, University of Alabama at Birmingham, Birmingham, AL 35294, USA. 
nuclei interacting via Coulomb potentials. The electrons are vectors in the positive energy subspace of the free Dirac operator and their kinetic energy is described by this operator. For a physical justification of this model see the papers of Sucher [8, 9, for applications of the model in computational atomic physics and quantum chemistry see Ishikawa and Koc 10, 11], and Pyykkö [12]. The Brown-Ravenhall Hamiltonian yields stability for sufficiently small values of the fine structure constant and the charge of the nuclei [13, 14, 15, 16, 3]; there are further rigorous results concerning the virial theorem [17] and eigenvalue estimates [18].

We are interested in the minimal energy of this model when it is corrected to account for the interaction of the electrons with the self-generated magnetic field. This correction may be done for instance by introducing a external magnetic field $\nabla \times \boldsymbol{A}$ to which the electrons are then minimally coupled and whose field energy is added to the energy of the system. The field $\boldsymbol{A}$ is now considered part of the system and hence the energy is to be minimized w.r.t. $\boldsymbol{A}$ as well. The minimizing $\boldsymbol{A}$ for a given electronic state is the self-generated one (to avoid instability for trivial reasons the gauge of $\boldsymbol{A}$ has to be fixed). The energy of this system is unbounded from below if $N \alpha^{3 / 2}$ is large, $\alpha$ being the fine structure constant, even if the vector potential is restricted to lie in a two parameter class $\left\{\gamma \boldsymbol{A}_{0}(\delta \boldsymbol{x}): \gamma, \delta \in \mathbb{R}_{+}\right\}$ where $\boldsymbol{A}_{0}$ is fixed and obeys a weak condition requiring not much more than $\boldsymbol{A}_{0} \not \equiv 0$. This is our first main result. It extends a previous result of Lieb et al. [3] and is reminiscent of the fact that a static non-vanishing classical magnetic field in QED is not regular, in the sense that the dressed electron-positron emission and absorption operators do not realize a representation of the CAR on the Fock space of the free field [19].

Alternatively the energy-shift due to the self-generated magnetic field may approximately be taken into account by including the Breit potential in the energy. The resulting model is unstable as well. That is, the energy is unbounded from below if $N \alpha^{3 / 2}$ is large, no matter how small $\alpha$ is. This is our second main result. It concerns a Hamiltonian that is closely related the Dirac-Coulomb-Breit or Dirac-Breit Hamiltonian, which is the bases for most calculations of relativistic effects in many electron atoms [8, 12]. We mention that for $\alpha=1 / 137$ the energy is bounded below if $N \leq 39$ and unbounded below if $N \geq 3.4 \cdot 10^{7}$ (Theorem 1 and Theorem 3).

A third way of accounting for the self-generated field is to couple the electrons to the quantized radiation field. From a simple argument using coherent states (Lemma 5) it follows that the instability of this model is rather worse than the instability of the model first discussed.

As mentioned above the instability with external magnetic field was previously found by Lieb et al. [3]. Our result extends their result and our proof is simpler. The model with Breit interaction corresponds to the classical system described by the Darwin Hamiltonian, which has been studied in the plasma physics literature (see [20] an the references therein). This classical model is thermodynamically unstable as well 21.

In Sections [1], III and IV] we introduce the models with external magnetic field, with Breit potential, and with quantized radiation field and prove their instability (Theorem 1 , Theorem 3, and Lemma 5). In Section III we also discuss dynamic nuclei for the model with Breit potential. There is an appendix where numerical values for stability bounds on $N \alpha^{3 / 2}$ given in the main text are computed.

\section{Instability with Classical Magnetic Field}

We begin with the model of matter with external magnetic field. For simplicity the electrons are assumed to be non-interacting and no nuclei are present. We could just as well treat a system of interacting electrons and static nuclei and would obtain essentially the same result (see Remark 4 below).

Consider a system of $N$ non-interacting electrons in the external magnetic field $\nabla \times \boldsymbol{A}$. 
The energy of this system is

$$
\mathcal{E}_{N}(\psi, \boldsymbol{A})=\left\langle\psi, \sum_{\mu=1}^{N} D_{\mu}(\boldsymbol{A}) \psi\right\rangle+\frac{1}{8 \pi} \int|\nabla \times \boldsymbol{A}(\boldsymbol{x})|^{2} d \boldsymbol{x}
$$

where $D_{\mu}(\boldsymbol{A})$ is the Dirac operator $D(\boldsymbol{A})=\boldsymbol{\alpha} \cdot\left(-i \nabla+\alpha^{1 / 2} \boldsymbol{A}(\boldsymbol{x})\right)+\beta m$ acting on the $\mu$-th particle, and the vector $\psi$, describing the state of the electrons, belongs to the Hilbert space

$$
\begin{aligned}
\mathcal{H}_{N} & =\bigwedge_{\mu=1}^{N} \Lambda_{+} L^{2}\left(\mathbb{R}^{3}, \mathbb{C}^{4}\right) \\
\Lambda_{+} & =\chi_{(0, \infty)}(D(\boldsymbol{A} \equiv 0)),
\end{aligned}
$$

or rather the dense subspace $\mathfrak{D}_{N}=\mathcal{H}_{N} \cap H^{1}\left[\left(\mathbb{R}^{3} \times\{1, \ldots, 4\}\right)^{N}\right]$. That is, an electron is by definition a vector in the positive energy subspace of the free Dirac operator. We will always assume the vector potential $\boldsymbol{A}$ belongs to the class $\mathcal{A}$ defined by the properties:

$$
\begin{array}{rlr}
\text { i) } & \nabla \cdot \boldsymbol{A}=0, \\
\text { ii) } & \boldsymbol{A}(\boldsymbol{x}) \rightarrow 0 \quad \text { as } \quad|\boldsymbol{x}| \rightarrow \infty, \\
\text { iii) } & \int_{\mathbb{R}^{3}}|\nabla \times \boldsymbol{A}|^{2}<\infty .
\end{array}
$$

Notice that $\mathcal{H}_{N}$ is not invariant under multiplication with smooth functions, in particular it is not invariant under gauge transformations of the states. It follows that the minimal energy for fixed $\boldsymbol{A}$ is gauge-dependent. It can actually be driven to $-\infty$ by a pure gauge transformation (see Remark 3 below). To avoid this trivial instability we fixed the gauge of $\boldsymbol{A}$ by imposing conditions i) and ii).

The constants $\alpha>0$ and $m \geq 0$ in the definition of $D(\boldsymbol{A})$ are the fine structure constant and the mass of the electron respectively. In our units $\hbar=1=c$, so that $\alpha=e^{2}$ which is about $1 / 137$ experimentally. We denote the Fourier transform of a function $f$ by $\widehat{f}$ or $\mathfrak{F}(f)$ and use $\boldsymbol{p}$ or $\boldsymbol{k}$ for its argument rather then $\boldsymbol{x}$ or $\boldsymbol{y}$. Our first result is

Theorem 1. Suppose $\boldsymbol{A} \in \mathcal{A}$ is such that $\operatorname{Re}[\boldsymbol{e} \cdot \widehat{\boldsymbol{A}}(\boldsymbol{p})]<0$ in $B(0, \varepsilon)$ for some $\boldsymbol{e} \in \mathbb{R}^{3}$ and $\varepsilon>0$. Then there exist a constant $C_{\boldsymbol{A}}$ such that for all $\alpha>0, m \geq 0$ and $N \geq C_{\boldsymbol{A}} \alpha^{-3 / 2}$

$$
\inf _{\psi \in \mathfrak{D}_{N},\|\psi\|=1, \gamma, \delta \in \mathbb{R}_{+}} \mathcal{E}_{N}(\psi, \gamma \boldsymbol{A}(\delta \boldsymbol{x}))=-\infty .
$$

Remarks.

1. It is sufficient that $\boldsymbol{A} \in \mathcal{A} \cap L^{1}$ and $\int_{\mathbb{R}^{3}} \boldsymbol{A}(\boldsymbol{x}) d \boldsymbol{x} \neq 0$, since $\widehat{\boldsymbol{A}}$ is then continuous and $\widehat{\boldsymbol{A}}(0) \neq 0$. Thus we have instability for virtually all non-vanishing $\boldsymbol{A} \in \mathcal{A}$.

2. The smallness of $N \alpha^{3 / 2}$ is not only necessary but also sufficient for stability (see [3, Section 4]).

3. If the condition ii) that $\boldsymbol{A}$ vanishes at infinity (and thus the gauge fixing) is dropped there is instability even for $N=1$ and the theorem becomes trivial. In fact for $N=1$ and $\boldsymbol{A}(\boldsymbol{x}) \equiv \boldsymbol{a} \neq 0, \mathcal{E}_{N=1}(\psi, \gamma \boldsymbol{A})=\langle\psi, D(0) \psi\rangle+\gamma \alpha^{1 / 2} \boldsymbol{a} \int \psi^{+}(\boldsymbol{x}) \boldsymbol{\alpha} \psi(\boldsymbol{x}) d \boldsymbol{x}$ which, as a function of $\gamma$, is unbounded from below for suitable $\psi \in \Lambda_{+} L^{2}\left(\mathbb{R}^{3}, \mathbb{C}^{4}\right)$.

4. The statement of the theorem also holds for the system of electrons and static nuclei with energy $\mathcal{E}_{N}(\psi, \boldsymbol{A})+\alpha\left\langle\psi, V_{c} \psi\right\rangle$ where

$$
V_{c}:=-\sum_{\mu=1}^{N} \sum_{\kappa=1}^{K} \frac{Z_{\kappa}}{\left|\boldsymbol{x}_{\mu}-\boldsymbol{R}_{\kappa}\right|}+\sum_{\mu<\nu}^{N} \frac{1}{\left|\boldsymbol{x}_{\mu}-\boldsymbol{x}_{\nu}\right|}+\sum_{\kappa<\sigma}^{K} \frac{Z_{\kappa} Z_{\sigma}}{\left|\boldsymbol{R}_{\kappa}-\boldsymbol{R}_{\sigma}\right|}
$$


if both $N$ and $\sum Z_{\kappa}$ are bigger than $C_{\boldsymbol{A}} \alpha^{-3 / 2}$ and if the energy is in addition minimized with respect to the pairwise distinct nuclear positions $\boldsymbol{R}_{\kappa}$. (see the proof of Theorem 3).

5. Quantizing the radiation field does not improve the stability of the system (see Section [V]).

The only way to restore stability we know is to replace $\mathcal{H}_{N}$ by the $\boldsymbol{A}$-dependent Hilbert space

$$
\mathcal{H}_{N, \boldsymbol{A}}=\bigwedge_{\mu=1}^{N} \chi_{(0, \infty)}(D(\boldsymbol{A})) L^{2}\left(\mathbb{R}^{3}, \mathbb{C}^{4}\right) .
$$

Obviously $\mathcal{E}_{N}(\psi, \boldsymbol{A}) \geq 0$ for $\psi \in \mathcal{H}_{N, \boldsymbol{A}}$. In fact even $\mathcal{E}_{N}(\psi, \boldsymbol{A})+\alpha\left\langle\psi, V_{c} \psi\right\rangle$ is non-negative for $Z_{\kappa}$ and $\alpha$ small enough [3].

Proof of Theorem 1 . We will only work with Slater determinants and the following representation of one-particle orbitals. If $u \in L^{2}\left(\mathbb{R}^{3} ; \mathbb{C}^{2}\right)$ then

$$
\widehat{\psi}(\boldsymbol{p})=\left(\frac{E(\boldsymbol{p})+m}{2 E(\boldsymbol{p})}\right)^{1 / 2}\left(\begin{array}{c}
u(\boldsymbol{p}) \\
\frac{\boldsymbol{\sigma} \cdot \boldsymbol{p}}{E(\boldsymbol{p})+m} u(\boldsymbol{p})
\end{array}\right),
$$

with $E(\boldsymbol{p})=\sqrt{\boldsymbol{p}^{2}+m^{2}}$, is the Fourier transform of a vector $\psi \in \Lambda_{+} L^{2}$, and the map $u \mapsto \psi, L^{2}\left(\mathbb{R}^{3} ; \mathbb{C}^{2}\right) \rightarrow \Lambda_{+} L^{2}\left(\mathbb{R}^{3} ; \mathbb{C}^{4}\right)$ is unitary.

It suffices to consider the case $m=0$ and find a Slater determinant $\psi=\psi_{1} \wedge \ldots \wedge \psi_{N}$ and $\gamma, \delta \in \mathbb{R}_{+}$such that $\mathcal{E}_{N}(\psi, \gamma \boldsymbol{A}(\delta \boldsymbol{x}))<0$. In fact by the scaling $\psi \mapsto \psi_{\delta}, \boldsymbol{A} \mapsto \boldsymbol{A}_{\delta}$ defined by $u_{\mu, \delta}=\delta^{-3 / 2} u_{\mu}\left(\delta^{-1} \boldsymbol{p}\right)$ and $\boldsymbol{A}_{\delta}(\boldsymbol{x})=\delta \boldsymbol{A}(\delta \boldsymbol{x})$ we can then drive the energy with $m>0$ to $-\infty$ because $\mathcal{E}\left(\psi_{\delta}, \boldsymbol{A}_{\delta}, m\right)=\delta \mathcal{E}(\psi, \boldsymbol{A}, m / \delta)$ and $\mathcal{E}(\psi, \boldsymbol{A}, m / \delta) \rightarrow \mathcal{E}(\psi, \boldsymbol{A}, m=0)$ for $\delta \rightarrow \infty$.

Choice of $\psi$. Let $Q$ be the unit cube $\left\{\boldsymbol{p} \in \mathbb{R}^{3} \mid 0 \leq p_{i} \leq 1\right\}, u(\boldsymbol{p})=\left(\chi_{Q}(\boldsymbol{p}), 0\right)^{T}$, and $\boldsymbol{e} \in \mathbb{R}^{3}$ an arbitrary unit vector. Set

$$
u_{\mu}(\boldsymbol{p})=u\left(\boldsymbol{p}-\lambda N^{1 / 3} \boldsymbol{e}-\boldsymbol{n}_{\mu}\right), \quad \mu=1, \ldots, N
$$

where $\lambda$ is a positive constant to be chosen sufficiently large later on, and $\left(\boldsymbol{n}_{\mu}\right)_{\mu=1 \ldots N} \subset \mathbb{Z}^{3}$ are the $N$ lattice sites nearest to the origin, i.e., $\max _{\mu=1 \ldots N}\left|\boldsymbol{n}_{\mu}\right|$ is minimal. We define $\psi=\psi_{1} \wedge \ldots \wedge \psi_{N}$ by

$$
\widehat{\psi}_{\mu}(\boldsymbol{p})=\frac{1}{\sqrt{2}}\left(\begin{array}{c}
u_{\mu}(\boldsymbol{p}) \\
\boldsymbol{\sigma} \cdot \boldsymbol{\omega}_{\boldsymbol{p}} u_{\mu}(\boldsymbol{p})
\end{array}\right), \quad \boldsymbol{\omega}_{\boldsymbol{p}}=\frac{\boldsymbol{p}}{|\boldsymbol{p}|},
$$

which is (2) for $m=0$. Then $\psi \in \mathcal{H}_{N}$ and $\left\langle\psi_{\mu}, \psi_{\nu}\right\rangle=\left\langle u_{\mu}, u_{\nu}\right\rangle=\delta_{\mu \nu}$. Notice that

$$
\left|\boldsymbol{p}-\lambda N^{1 / 3} \boldsymbol{e}\right| \leq N^{1 / 3} \quad \text { for all } \quad \boldsymbol{p} \in \operatorname{supp}\left(u_{\mu}\right)
$$

at least for large $N$ (see the appendix), i.e., in Fourier space all electrons are localized in a ball with radius $N^{1 / 3}$ and a distance from the origin which is large compared to the radius (since $\lambda$ will be large).

Since $\psi=\psi_{1} \wedge \ldots \wedge \psi_{N}$ and $m>0$ we have

$$
\begin{aligned}
\mathcal{E}_{N}(\psi, \boldsymbol{A})= & \sum_{\mu=1}^{N}\left\langle\psi_{\mu},|\nabla| \psi_{\mu}\right\rangle+\alpha^{1 / 2} \sum_{\mu=1}^{N} \int \boldsymbol{J}_{\mu}(\boldsymbol{x}) \boldsymbol{A}(\boldsymbol{x}) d \boldsymbol{x} \\
& +\frac{1}{8 \pi} \int|\nabla \times \boldsymbol{A}(\boldsymbol{x})|^{2} d \boldsymbol{x}
\end{aligned}
$$


where $\boldsymbol{J}_{\mu}(\boldsymbol{x})=\psi_{\mu}^{*}(x) \boldsymbol{\alpha} \psi_{\mu}(x)$. By definition of $\psi_{\mu}$

$$
\widehat{\boldsymbol{J}}_{\mu}(\boldsymbol{p})=\frac{1}{2}(2 \pi)^{-3 / 2} \int u_{\mu}^{*}(\boldsymbol{k}-\boldsymbol{p})\left[\boldsymbol{\sigma}\left(\boldsymbol{\omega}_{\boldsymbol{k}} \cdot \boldsymbol{\sigma}\right)+\left(\boldsymbol{\omega}_{\boldsymbol{k}-\boldsymbol{p}} \cdot \boldsymbol{\sigma}\right) \boldsymbol{\sigma}\right] u_{\mu}(\boldsymbol{k}) d \boldsymbol{k} .
$$

Replace here $u_{\mu}$ by its defining expression and substitute $\left(\boldsymbol{k}-\lambda N^{1 / 3} \boldsymbol{e}-\boldsymbol{n}_{\mu}\right) \mapsto \boldsymbol{k}$. Since $\boldsymbol{\omega}_{\boldsymbol{k}+\lambda N^{1 / 3} \boldsymbol{e}+\boldsymbol{n}_{\mu}} \rightarrow \boldsymbol{e}$ as $\lambda \rightarrow \infty$ and since $u$ has compact support, it follows that $\widehat{\boldsymbol{J}}_{\mu}(\boldsymbol{p})$ converges to the current

$$
\widehat{\boldsymbol{J}}_{0}(\boldsymbol{p})=\boldsymbol{e}(2 \pi)^{-2 / 3} \int u^{*}(\boldsymbol{k}-\boldsymbol{p}) u(\boldsymbol{k}) d \boldsymbol{k}
$$

as $\lambda \rightarrow \infty$. More precisely $\left|\widehat{\boldsymbol{J}}_{\mu}(\boldsymbol{p})-\widehat{\boldsymbol{J}}_{0}(\boldsymbol{p})\right| \leq C \lambda^{-1}\left|\widehat{\boldsymbol{J}}_{0}(\boldsymbol{p})\right|$ for $\lambda \geq \lambda_{0}$ where $\lambda_{0}$ and $C$ are independent of $\mu$ and $N$. From $\widehat{\boldsymbol{J}}_{0}(\boldsymbol{p})|\boldsymbol{p}|^{-1}, \widehat{\boldsymbol{A}}(\boldsymbol{p})|\boldsymbol{p}| \in L^{2}$ it follows that

$$
\int \widehat{\boldsymbol{J}}_{\mu}^{*}(\boldsymbol{p}) \widehat{\boldsymbol{A}}(\boldsymbol{p}) d \boldsymbol{p}=\int \widehat{\boldsymbol{J}}_{0}^{*}(\boldsymbol{p}) \widehat{\boldsymbol{A}}(\boldsymbol{p}) d \boldsymbol{p}+O\left(\lambda^{-1}\right), \quad \lambda \rightarrow \infty .
$$

After a scaling $\boldsymbol{A} \mapsto \boldsymbol{A}_{\delta}$ we may assume $\operatorname{Re}[\boldsymbol{e} \cdot \widehat{\boldsymbol{A}}(\boldsymbol{p})]<0$ in the support of $\widehat{\boldsymbol{J}}_{0}$ rather then in $B(0, \varepsilon)$, so that (9) is bounded from above by some $-c_{1}<0$ for $\lambda \geq \lambda_{0}$ where $c_{1}$ and $\lambda_{0}$ are independent of $\mu$ and $N$. Observing finally that

$$
\left\langle\psi_{\mu},|\nabla| \psi_{\mu}\right\rangle=\int\left|\widehat{\psi}_{\mu}(\boldsymbol{p})\right|^{2}|\boldsymbol{p}| d \boldsymbol{p} \leq(\lambda+1) N^{1 / 3}
$$

for all $\mu$, we conclude

$$
\begin{aligned}
\mathcal{E}_{N}(\psi, \gamma \boldsymbol{A}) & \leq\left(\lambda_{0}+1\right) N^{4 / 3}-\alpha^{1 / 2} \gamma N c_{1}+\gamma^{2} c_{2} \\
& =\left(\lambda_{0}+1\right) N^{4 / 3}-\alpha \frac{c_{1}^{2}}{4 c_{2}} N^{2}
\end{aligned}
$$

which is negative for $N \alpha^{3 / 2}$ large enough. At the end we inserted the optimal $\gamma$.

The theorem has the obvious corollary

Corollary 2. There is a constant $C$ such that for all $\alpha>0, m \geq 0$ and $N \geq C \alpha^{-3 / 2}$,

$$
\inf _{\psi \in \mathfrak{D}_{N},\|\psi\|=1 ; \boldsymbol{A} \in \mathcal{A}} \mathcal{E}_{N}(\psi, \boldsymbol{A})=-\infty .
$$

This result is due to Lieb, Siedentop, and Solovej [3].

Remark. It is sufficient that $C=1.4 \cdot 10^{5}$ or that $N \geq 3.4 \cdot 10^{7}$ for $\alpha^{-1}=137$, see the appendix.

To conclude this section we compute $\min _{\boldsymbol{A} \in \mathcal{A}} \mathcal{E}_{N}(\psi, \boldsymbol{A})$. This will provide a link to the instability with Breit-potential discussed in the next section. To exhibit the $\boldsymbol{A}$-dependence we write the energy as

$$
\mathcal{E}_{N}(\psi, \boldsymbol{A})=\mathcal{E}_{N}(\psi, \boldsymbol{A} \equiv 0)+\alpha^{1 / 2} \int \boldsymbol{J}(\boldsymbol{x}) \boldsymbol{A}(\boldsymbol{x})+\frac{1}{8 \pi} \int|\nabla \times \boldsymbol{A}(\boldsymbol{x})|^{2} d \boldsymbol{x},
$$

where $\boldsymbol{J}(\boldsymbol{x})$ is the probability current density associated with $\psi$. Its functional dependence on $\psi$ is not crucial here. A straight forward computation shows that the Euler-Lagrange equation for $\boldsymbol{A}$ is $-\Delta \boldsymbol{A}=4 \pi \alpha^{1 / 2} \boldsymbol{J}_{T}$ where $\boldsymbol{J}_{T}$ is the divergence free - or transversal - part of $\boldsymbol{J}$. Comparing this equation with the Maxwell-equation for $\boldsymbol{A}$ in Coulomb gauge, which is $\square \boldsymbol{A}=4 \pi \alpha^{1 / 2} \boldsymbol{J}_{T}$, we find that the minimizing magnetic field is the self-generated one up to effects of retardation. Solving the Euler-Lagrange equation gives

$$
\min _{\boldsymbol{A} \in \mathcal{A}} \mathcal{E}_{N}(\psi, \boldsymbol{A})=\mathcal{E}_{N}(\psi, \boldsymbol{A} \equiv 0)-\frac{\alpha}{2} \int \frac{\boldsymbol{J}_{T}(\boldsymbol{x}) \boldsymbol{J}_{T}(\boldsymbol{y})}{|\boldsymbol{x}-\boldsymbol{y}|} d \boldsymbol{x} d \boldsymbol{y} .
$$




\section{Instability with Breit Potential}

\section{III.A Static nuclei}

We now consider a system of $N$ (interacting) electrons in the external electric field of $K$ static nuclei. There is no external magnetic field but a self-generated one which is approximately accounted for by the Breit potential. The energy is now

$$
\mathcal{E}_{N}(\psi, \boldsymbol{R})=\left\langle\psi,\left(\sum_{\mu=1}^{N} D_{\mu}+\alpha\left(V_{c}-B\right)\right) \psi\right\rangle
$$

where

$$
B=\sum_{\mu<\nu}^{N} \frac{1}{2\left|\boldsymbol{x}_{\mu}-\boldsymbol{x}_{\nu}\right|}\left(\sum_{i} \alpha_{i, \mu} \otimes \alpha_{i, \nu}+\frac{\boldsymbol{\alpha}_{\mu} \cdot\left(\boldsymbol{x}_{\mu}-\boldsymbol{x}_{\nu}\right) \otimes \boldsymbol{\alpha}_{\nu} \cdot\left(\boldsymbol{x}_{\mu}-\boldsymbol{x}_{\nu}\right)}{\left|\boldsymbol{x}_{\mu}-\boldsymbol{x}_{\nu}\right|^{2}}\right)
$$

and $V_{c}$ is the Coulomb potential defined in (11). $\boldsymbol{R}$ denotes the $K$-tuple $\left(\boldsymbol{R}_{1}, \ldots, \boldsymbol{R}_{K}\right)$ of pairwise different nuclear positions and $D_{\mu}=D_{\mu}(\boldsymbol{A} \equiv 0)$. As before $\psi$ belongs to $\mathfrak{D}_{N} \subset \mathcal{H}_{N}$. The interaction $-\alpha B$ is usually derived from the corresponding interaction in the Darwin Hamiltonian by the quantization $\boldsymbol{p} / m \mapsto \boldsymbol{\alpha}$ [22] or from QED: treating the interactions of the electrons with the quantized radiation field in second order perturbation theory leads to a shift of the bound state energy levels approximately given by $-\alpha\langle\psi, B \psi\rangle$ [23]. Important for our purpose is that

$$
\langle\psi, B \psi\rangle+\left(\begin{array}{c}
\text { self-energy \& } \\
\text { exchange terms }
\end{array}\right)=\frac{1}{2} \int \frac{\boldsymbol{J}_{T}(\boldsymbol{x}) \boldsymbol{J}_{T}(\boldsymbol{y})}{|\boldsymbol{x}-\boldsymbol{y}|} d \boldsymbol{x} d \boldsymbol{y}
$$

for any Slater determinant $\psi=\psi_{1} \wedge \ldots \wedge \psi_{N}$ of orthonormal functions $\psi_{\mu}$ (see the proof of Theorem 31).

We are interested in the lowest possible energy

$$
E_{N, K}=\inf \mathcal{E}_{N}(\psi, \boldsymbol{R})
$$

where the infimum is taken over all $\psi \in \mathfrak{D}_{N}$ with $\|\psi\|=1$ and all $K$-tuples $\left(\boldsymbol{R}_{1}, \ldots, \boldsymbol{R}_{K}\right)$ with $\boldsymbol{R}_{j} \neq \boldsymbol{R}_{k}$ for $j \neq k$. Our second main result is

Theorem 3. There exists a constant $C$ such that for all $\alpha>0, m \geq 0, K \in \mathbb{N}$ and $Z_{1}, \ldots, Z_{K} \in \mathbb{R}_{+}$

$$
E_{N, K}=-\infty
$$

whenever $N, \sum Z_{\kappa} \geq C \max \left(\alpha^{-3 / 2}, 1\right)$. If $\sum Z_{\kappa}^{2} \geq 1$ it suffices that $C=5 \cdot 10^{4}$ or - when $\alpha^{-1}=137$ - that $N=\sum Z_{\kappa} \geq 3.4 \cdot 10^{7}$.

Remarks.

1. Similar as in Section If $V_{c}$ and hence the condition on $\sum Z_{\kappa}$ may be dropped. Then there is instability for $N \geq C \max \left(\alpha^{-3 / 2}, 1\right)$. It is for completeness of the model we keep $V_{c}$ in this section.

2. Without $B$ the energy is proven to be non-negative $\alpha Z_{\kappa} \leq 2 / \pi$ for all $\kappa$ and if $\alpha \leq 1 / 94$ [6] (see also [3]). One expects however stability even for $\alpha Z_{\kappa} \leq 2\left(\frac{2}{\pi}+\frac{\pi}{2}\right)^{-1} \alpha \leq 0.12$ [13, 16], which would cover the atomic numbers of all known elements.

At least partly this theorem can be understood from Corollary 2, Equation (11) and Equation (14). 
Proof of Theorem 8. To begin with we prove (14). Let $\psi=\psi_{1} \wedge \ldots \wedge \psi_{N}$ with $\left\langle\psi_{\mu}, \psi_{\nu}\right\rangle=\delta_{\mu \nu}$ and let $\boldsymbol{J}(\boldsymbol{x})=\sum_{\mu=1}^{N} \psi_{\mu}^{+}(x) \boldsymbol{\alpha} \psi_{\mu}(x)$ be the current density of $\psi$. Note that $\widehat{J}_{T, i}(\boldsymbol{p})=$ $\sum_{j=1}^{3}\left(\delta_{i j}-\frac{p_{i} p_{j}}{p^{2}}\right) \widehat{J}_{j}(\boldsymbol{p})$ and that

$$
\mathfrak{F} \frac{4 \pi}{p^{2}}\left(\delta_{i j}-\frac{p_{i} p_{j}}{p^{2}}\right)=\frac{1}{2|x|}\left(\delta_{i j}+\frac{x_{i} x_{j}}{x^{2}}\right) .
$$

With $B(\boldsymbol{x})$ defined by

$$
B(x)=\frac{1}{2|x|} \sum_{i, j} \alpha_{i}\left(\delta_{i j}+\frac{x_{i} x_{j}}{x^{2}}\right) \alpha_{j}=\frac{1}{2|x|}\left(\sum_{i} \alpha_{i} \otimes \alpha_{i}+\frac{\boldsymbol{\alpha} \cdot \boldsymbol{x} \otimes \boldsymbol{\alpha} \cdot \boldsymbol{x}}{|\boldsymbol{x}|^{2}}\right)
$$

it follows that

$$
\begin{aligned}
\frac{1}{2} \int \frac{\boldsymbol{J}_{T}(\boldsymbol{x}) \boldsymbol{J}_{T}(\boldsymbol{y})}{|\boldsymbol{x}-\boldsymbol{y}|} d \boldsymbol{x} d \boldsymbol{y} & =\frac{1}{2} \sum_{\mu, \nu}\left\langle\psi_{\mu} \otimes \psi_{\nu}, B(x-y) \psi_{\mu} \otimes \psi_{\nu}\right\rangle \\
& =\langle\psi, B \psi\rangle+\frac{1}{2} \sum_{\mu, \nu}\left\langle\psi_{\mu} \otimes \psi_{\nu}, B(x-y) \psi_{\nu} \otimes \psi_{\mu}\right\rangle .
\end{aligned}
$$

which is equation (14). Similar as in the proof of Theorem 1 it suffices to consider the case $m=0$ and to find a Slater determinant $\psi=\psi_{1} \wedge \ldots \wedge \psi_{N}$ and nuclear positions $\boldsymbol{R}_{1}, \ldots, \boldsymbol{R}_{K}$ such that $\mathcal{E}_{N}(\psi, \boldsymbol{R})<0$.

Choice of the nuclear positions. A beautiful argument given in [3] show that, after moving some electrons or nuclei far away from all others

$$
\left\langle\psi, V_{c} \psi\right\rangle \leq \varepsilon+\frac{1}{2 N^{2}} \sum_{\mu, \nu} \int \frac{\left|\psi_{\mu}(\boldsymbol{x})\right|^{2}\left|\psi_{\nu}(\boldsymbol{y})\right|^{2}}{|\boldsymbol{x}-\boldsymbol{y}|} d \boldsymbol{x} d \boldsymbol{y}
$$

for suitably chosen nuclear positions. Here $\varepsilon>0$ is the (arbitrary small) contribution of the particles moved away. The second term can be dropped if $\sum_{\kappa=1}^{K} Z_{\kappa}^{2} \geq 1$. We use the inequality obtained in [14 to estimate it from above and find

$$
\left\langle\psi, V_{c} \psi\right\rangle \leq \varepsilon+\operatorname{const} \frac{1}{N} \sum_{\mu=1}^{N}\left\langle\psi_{\mu}, D \psi_{\mu}\right\rangle
$$

The number $N$ of remaining electrons obeys $N<\sum Z_{\kappa}+1$ which is the reason for the assumption on $\sum Z_{\kappa}$. Of course the choice of the nuclear positions depends on $\psi$, which has not been specified yet.

Define one-particle orbitals $\psi_{\mu}$ and currents $\boldsymbol{J}_{\mu}$ and $\boldsymbol{J}_{0}$ exactly as in the proof of Theorem 1 with $\boldsymbol{e}$ being an arbitrary unit vector in $\mathbb{R}^{3}$. The convergence $\widehat{\boldsymbol{J}}_{\mu}(\boldsymbol{p}) \rightarrow \widehat{\boldsymbol{J}}_{0}(\boldsymbol{p})$ as $\lambda \rightarrow \infty$ now implies that

$$
\begin{aligned}
\frac{1}{2} \int \frac{\boldsymbol{J}_{T}(\boldsymbol{x}) \boldsymbol{J}_{T}(\boldsymbol{y})}{|\boldsymbol{x}-\boldsymbol{y}|} d \boldsymbol{x} d \boldsymbol{y} & =N^{2}\left[\frac{1}{2} \int \frac{\boldsymbol{J}_{0, T}(\boldsymbol{x}) \boldsymbol{J}_{0, T}(\boldsymbol{y})}{|\boldsymbol{x}-\boldsymbol{y}|} d \boldsymbol{x} d \boldsymbol{y}+O\left(\lambda^{-1}\right)\right] \\
& \geq c_{2} N^{2}
\end{aligned}
$$

for $\lambda \geq \lambda_{0}$, where $\lambda_{0}$ and $c_{2}>0$ are independent of $N$.

To estimate the sum of exchange- and self-energy terms in (15) notice that

$$
\left\langle\psi_{\mu} \otimes \psi_{\nu}, B(x-y) \psi_{\nu} \otimes \psi_{\mu}\right\rangle=\int \frac{4 \pi}{p^{2}}\left|\widehat{\boldsymbol{J}}_{\mu \nu, T}(\boldsymbol{p})\right|^{2} d \boldsymbol{p},
$$


where $\boldsymbol{J}_{\mu \nu}(\boldsymbol{x})=\psi_{\mu}^{*}(x) \boldsymbol{\alpha} \psi_{\nu}(x)$. After writing $\widehat{\boldsymbol{J}}_{\mu \nu}(\boldsymbol{p})$ as an integral in Fourier space in terms of $u_{\mu}$ and $u_{\nu}$ similar as in (7) it is easily seen, using the support properties of $u_{\mu}$ and $u_{\nu}$, that

$$
\left|\widehat{\boldsymbol{J}}_{\mu \nu, T}(\boldsymbol{p})\right|^{2} \leq\left|\widehat{\boldsymbol{J}}_{\mu \nu}(\boldsymbol{p})\right|^{2} \leq 3(2 \pi)^{-3} \chi\left(\left|\boldsymbol{p}+\boldsymbol{n}_{\mu}-\boldsymbol{n}_{\nu}\right| \leq \sqrt{3}\right) .
$$

The $N$ balls $B\left(\boldsymbol{n}_{\nu}, \sqrt{3}\right), \nu=1, \ldots, N$ all lie in the ball $B\left(0, N^{1 / 3}\right)$ and cover a given point at most, say, $4^{3}=64$ times (replace the balls by cubes with side $2 \sqrt{3}$ ). Therefore $(19)$ implies

$$
\sum_{\nu=1}^{N}\left|\widehat{\boldsymbol{J}}_{\mu \nu}(\boldsymbol{p})\right|^{2} \leq 192(2 \pi)^{-3} \chi\left(\left|\boldsymbol{p}+\boldsymbol{n}_{\mu}\right|<N^{1 / 3}\right) \leq \frac{24}{\pi^{3}} \chi\left(|\boldsymbol{p}|<2 N^{1 / 3}\right)
$$

which in conjunction with (18) gives

$$
\frac{1}{2} \sum_{\mu, \nu}\left\langle\psi_{\mu} \otimes \psi_{\nu}, B(x-y) \psi_{\nu} \otimes \psi_{\mu}\right\rangle \leq \frac{384}{\pi} N^{4 / 3} .
$$

Rewriting the energy using (15) and inserting the estimates (16), (10), (17) and (20) we arrive at

$$
\mathcal{E}_{N}(\psi, \boldsymbol{R}) \leq c_{1}(1+\alpha) N^{4 / 3}-c_{2} \alpha N^{2}, \quad c_{2}>0
$$

which is negative for $N>$ const $\max \left(\alpha^{-3 / 2}, 1\right)$. This proves the theorem.

For small $N$ and small $\alpha$ there is stability. A similar result for the energy in Section 1 was proved in [3].

Theorem 4. Suppose $\tilde{\alpha} \leq 1 / 94, \max _{\kappa} Z_{\kappa} \leq 2 / \pi \tilde{\alpha}^{-1}$ and $N-1 \leq 2(2 / \pi+\pi / 2)\left(\alpha^{-1}-\tilde{\alpha}^{-1}\right)$. Then $E_{N, K} \geq 0$. Inserting $\tilde{\alpha}=1 / 94$ and $\alpha=1 / 137$ we find stability for $N \leq 39$ and $\max Z_{\kappa} \leq 59$.

Proof. Since $B(x) \leq 2 /|x|$ on $\mathbb{C}^{4} \otimes \mathbb{C}^{4}$ and $1 /|x| \leq \delta^{-1} D$ on $\Lambda_{+} L^{2}\left(\mathbb{R}^{3} ; \mathbb{C}^{4}\right)$ where $\delta=$ $2(2 / \pi+\pi / 2)$ [14 one has by the symmetry property of the states in $\mathcal{H}_{N}$

$$
B \leq \frac{N-1}{\delta} \sum_{\mu=1}^{N} D_{\mu} \quad \text { on } \mathcal{H}_{N} .
$$

Furthermore

$$
V_{c} \geq-\frac{1}{\tilde{\alpha}} \sum_{\mu=1}^{N} D_{\mu} \quad \text { on } \mathcal{H}_{N}
$$

for all $\tilde{\alpha}>0$ with $\tilde{\alpha} \max Z_{\kappa} \leq \frac{2}{\pi}$ and $\tilde{\alpha} q \leq 1 / 47$ by [6], where the number $q$ of spin states may be set equal 2 [3]. Inserting (21) and (22) in the energy proves the theorem.

\section{III.B Dynamic nuclei}

Making the nuclei dynamical would improve stability if their kinetic energy were the only term we added to (12). However if the nuclei are relativistic spin $1 / 2$ particles like the electrons and if the Breit-potential couples all pairs of particles, taking their charges into account, then the instability will actually become worse.

Let us illustrate this for a system of $N$ electrons and $K$ identical nuclei of spin $1 / 2$ and atomic number $Z>0$. These nuclei are described by vectors in the positive energy subspace 
of the free Dirac operator with the mass $M>0$ of the nuclei. To prove instability we adopt the strategy of the proof of Theorem 3 and thus assume $M=0$ and $m=0$. As a trial-wave function we take

$$
\psi=\left(\psi_{1} \wedge \ldots \wedge \psi_{N}\right) \otimes\left(\phi_{1} \wedge \ldots \wedge \phi_{K}\right)
$$

where $\psi_{\mu}$ is defined by equations (3) and (位) and $\phi_{\kappa}$ is defined like $\psi_{\kappa}$ except that $\boldsymbol{e}$ and $N$ are replaced by $-\boldsymbol{e}$ and $K$ respectively. It follows that in the limit $\lambda \rightarrow \infty$ we get $N+K$ (charge-) currents, the nuclear ones being larger than the electronic ones by a factor of $Z$ but otherwise identical. The Breit interactions thus gives a negative contribution to the energy of order $\alpha(N+Z K)^{2}$. While the parallel currents of the $N+K$ particles add up, the opposite charges of the electrons and nuclei cancel themselves. In fact for $\psi$ defined as above

$$
\begin{aligned}
\left\langle\psi, V_{c} \psi\right\rangle \leq & \sum_{\mu<\nu}^{N} \int d \boldsymbol{x} d \boldsymbol{y} \frac{\left|\psi_{\mu}(\boldsymbol{x})\right|^{2}\left|\psi_{\nu}(\boldsymbol{y})\right|^{2}}{|\boldsymbol{x}-\boldsymbol{y}|} \\
& +Z^{2} \sum_{\kappa<\sigma}^{K} \int d \boldsymbol{R}_{1} d \boldsymbol{R}_{2} \frac{\left|\phi_{\kappa}\left(\boldsymbol{R}_{1}\right)\right|^{2}\left|\phi_{\sigma}\left(\boldsymbol{R}_{2}\right)\right|^{2}}{\left|\boldsymbol{R}_{1}-\boldsymbol{R}_{2}\right|} \\
& +Z \sum_{\kappa=1}^{K} \sum_{\mu=1}^{N} \int d \boldsymbol{x} d \boldsymbol{R} \frac{\left|\psi_{\mu}(\boldsymbol{x})\right|^{2}\left|\phi_{\kappa}(\boldsymbol{R})\right|^{2}}{|\boldsymbol{x}-\boldsymbol{R}|} \\
= & {\left[\frac{N(N-1)}{2}+Z^{2} \frac{K(K-1)}{2}-N K Z\right]\left(I+O\left(\lambda^{-1}\right)\right) } \\
= & {\left[(K Z-N)^{2}-K Z^{2}-N\right]\left(I / 2+O\left(\lambda^{-1}\right)\right), }
\end{aligned}
$$

where $I$ is the limit of the above double integrals as $\lambda \rightarrow \infty$. Hence $\left\langle\psi, V_{c} \psi\right\rangle$ is negative, e.g., if $K Z=N$ and $\lambda$ is large. To achieve this in the static case we had to choose the nuclear positions properly. It is instructive to recall how this was done. The total energy is bounded from above by $c_{1}\left(N^{4 / 3}+K^{4 / 3}\right)-c_{2} \alpha(N+K Z)^{2}, c_{2}>0$, for $N=K Z$ and $\lambda$ large, and is therefore negative for $N=K Z$ large enough.

\section{Stability and Instability with Quantized Radiation Field}

Instability for the model with classical magnetic field implies instability for the model with quantized radiation field without UV-cutoff. In fact, for each classical magnetic field there is a coherent state of photons which reproduces the classical field as far as the energy is concerned. If an UV cutoff is introduced the relativistic scale invariance of the energy is broken and stability of the first kind is restored. The lower bound depends on the cutoff and goes to $-\infty$ as the cutoff is removed.

The state of the system is now described by a vector $\Psi \in \mathcal{H}_{N} \otimes \mathcal{F}$ where $\mathcal{F}$ denotes the bosonic Fock-space over $L^{2}\left(\mathbb{R}^{3}\right) \otimes \mathbb{C}^{2}$, the factor $\mathbb{C}^{2}$ accounting for the two possible polarizations of the transversal photons, and the total energy of $\Psi$ is

$$
\begin{aligned}
\mathcal{E}_{N}^{\mathrm{qed}}(\Psi)= & \left\langle\Psi, \sum_{\mu=1}^{N}\left[\boldsymbol{\alpha}_{\mu} \cdot\left(-i \nabla_{\mu}+\alpha^{1 / 2} \boldsymbol{A}\left(\boldsymbol{x}_{\mu}\right)\right)+\beta_{\mu} m\right] \Psi\right\rangle \\
& +\left\langle\Psi,\left(1 \otimes H_{f}\right) \Psi\right\rangle \\
H_{f}= & \sum_{\lambda=1}^{2} \int d \boldsymbol{k}|\boldsymbol{k}| a_{\lambda}^{\dagger}(\boldsymbol{k}) a_{\lambda}(\boldsymbol{k}),
\end{aligned}
$$


where

$$
\begin{aligned}
\boldsymbol{A}(\boldsymbol{x}) & :=\sum_{\lambda=1}^{2} \int d k\left[\boldsymbol{e}_{\lambda}(\boldsymbol{k}) e^{i \boldsymbol{k} \boldsymbol{x}} \otimes a_{\lambda}(\boldsymbol{k})+\boldsymbol{e}_{\lambda}(\boldsymbol{k}) e^{-i \boldsymbol{k} \boldsymbol{x}} \otimes a_{\lambda}^{\dagger}(\boldsymbol{k})\right] \\
& =: \boldsymbol{A}^{+}(\boldsymbol{x})+\boldsymbol{A}^{+}(\boldsymbol{x})^{*}
\end{aligned}
$$

is the quantized vector potential in Coulomb gauge. The operators $a_{\lambda}(\boldsymbol{k})$ and $a_{\lambda}^{\dagger}(\boldsymbol{k})$ are creation and annihilation operators acting on $\mathcal{F}$ and obeying the CCR

$$
\left[a_{\lambda}\left(\boldsymbol{k}_{1}\right), a_{\mu}^{\dagger}\left(\boldsymbol{k}_{2}\right)\right]=\delta_{\mu \nu} \delta\left(\boldsymbol{k}_{1}-\boldsymbol{k}_{2}\right), \quad\left[a_{\lambda}^{\sharp}\left(\boldsymbol{k}_{1}\right), a_{\mu}^{\sharp}\left(\boldsymbol{k}_{2}\right)\right]=0
$$

where $a_{\lambda}^{\sharp}=a_{\lambda}$ or $a_{\lambda}^{\dagger}$, and the two polarization vectors $\boldsymbol{e}_{\lambda}(\boldsymbol{k})$ are orthonormal and perpendicular to $\boldsymbol{k}$ for each $\boldsymbol{k} \in \mathbb{R}^{3}$. We use $d k$ as a short hand for $(2 \pi)^{-3 / 2}(2|\boldsymbol{k}|)^{-1 / 2} d \boldsymbol{k}$, and the subindex of $\boldsymbol{\alpha}_{\mu}, \nabla_{\mu}$ and $\beta_{\mu}$ indicates that these one particle operators act on the $\mu$-th particle. While we used Gaussian units in Section II and III we now work with Heaviside Lorenz units.

Lemma 5. For each $\boldsymbol{A}_{c l} \in \mathcal{A} \cap L^{2}\left(\mathbb{R}^{3}\right)$ there exists a vector $\theta \in \mathcal{F}$ (coherent state) such that

$$
\mathcal{E}_{N}^{q e d}(\psi \otimes \theta)=\mathcal{E}_{N}\left(\psi, \boldsymbol{A}_{c l}\right)
$$

for all $\psi \in \mathfrak{D}_{N}$.

Proof. Pick $\boldsymbol{A}_{c l} \in \mathcal{A} \cap L^{2}\left(\mathbb{R}^{3}\right)$ and define $\eta_{\lambda(\boldsymbol{k})}=(|\boldsymbol{k}| / 2)^{1 / 2} \boldsymbol{e}_{\lambda}(\boldsymbol{k}) \cdot \widehat{\boldsymbol{A}}_{c l}(\boldsymbol{k})$ so that $\boldsymbol{A}_{c l}(\boldsymbol{x})=$ $\boldsymbol{A}_{c l}^{+}(\boldsymbol{x})+\boldsymbol{A}_{c l}^{+}(\boldsymbol{x})^{*}$ with

$$
\boldsymbol{A}_{c l}^{+}(\boldsymbol{x})=\sum_{\lambda=1}^{2} \int d k \eta_{\lambda}(\boldsymbol{k}) \boldsymbol{e}_{\lambda}(\boldsymbol{k}) e^{i \boldsymbol{k} \boldsymbol{x}}
$$

Next set

$$
\Pi(\eta):=i \sum_{\lambda=1}^{2} \int d \boldsymbol{k}\left[\overline{\eta_{\lambda}(\boldsymbol{k})} a_{\lambda}(\boldsymbol{k})+\eta_{\lambda}(\boldsymbol{k}) a_{\lambda}^{\dagger}(\boldsymbol{k})\right]
$$

and $\Theta=e^{-i \Pi(\eta)} \Omega \in \mathcal{F}$. $\Theta$ is called a coherent state, it is normalized and most importantly it is an eigenvector of all annihilation operators

$$
a_{\lambda}(\boldsymbol{k}) \Theta=\eta_{\lambda}(\boldsymbol{k}) \Theta .
$$

¿From (24), (25) and the definition of $\eta_{\lambda}(\boldsymbol{k})$ it follows that

$$
\boldsymbol{\alpha}_{\mu} \boldsymbol{A}^{+}\left(\boldsymbol{x}_{\mu}\right) \psi \otimes \Theta=\left(\boldsymbol{\alpha}_{\mu} \boldsymbol{A}_{c l}^{+}\left(\boldsymbol{x}_{\mu}\right) \otimes \mathbf{1}\right) \psi \otimes \Theta
$$

and

$$
\left\langle\Theta, H_{f} \Theta\right\rangle=\int d \boldsymbol{k}|\boldsymbol{k}| \sum_{\lambda}\left|\eta_{\lambda}(\boldsymbol{k})\right|^{2}=\frac{1}{2} \int d \boldsymbol{k} k^{2}\left|\widehat{\boldsymbol{A}}_{c l}(\boldsymbol{k})\right|^{2} .
$$

Inserting this in the energy proves the theorem.

If an ultraviolet cutoff is introduced in the field operator $\boldsymbol{A}(\boldsymbol{x})$ then stability of the first kind is restored for all $N$ and a certain range of values for $\alpha$ and $Z_{\kappa}$. This follows from 24 , Lemma I.5] and [3, Theorem 1]. 


\section{Acknowledgement}

It is a pleasure to thank Heinz Siedentop for many discussions, and Arne Jensen, Jan Philip Solovej and Erik Skibsted for the hospitality at Aarhus University in August 97, where this work was begun. M. G. also thanks Michael Loss for clarifying discussions. This work was partially supported by the European Union under grant ERB4001GT950214 and under the TMR-network grant FMRX-CT 96-0001.

\section{Appendix}

To obtain the numerical values for the constants in Corollary 2 and Theorem 3 we follow the proof of Theorem 3, up to a few modifications and explicitly evaluate the constants in this proof.

The main modifications are that the two-spinor $u$ is now defined in terms of the (normalized) characteristic function of the ball with radius $1 / 2$ contained in the unit cube $\left\{\boldsymbol{p} \mid 0 \leq p_{i} \leq 1\right\}$ and that the 4-spinors $\psi_{2 \mu-1}$ are defined in terms of the $\psi_{2 \mu}$ 's by interchanging the components of $\boldsymbol{u}$, while $\boldsymbol{n}_{2 \mu-1}$ runs over the $N / 2$ or - if $N$ is odd - the $(N+1) / 2$ lattice sites of $\mathbb{Z}^{3}$ closest to the origin. The balls simplify the computation of $\widehat{\boldsymbol{J}}_{0}(\boldsymbol{p})$ and the double occupation $\boldsymbol{n}_{2 \mu-1}=\boldsymbol{n}_{2 \mu}$ reduces the kinetic energy. To begin with we note that the $n$ unit cubes of the lattice $\mathbb{Z}^{3}$ which are closest to the origin, all fit in a ball of radius

$$
n^{1 / 3}\left(\frac{3}{4 \pi}\right)^{1 / 3}+\sqrt{3}
$$

In particular the $N / 2$ or $(N+1) / 2$ unit cubes containing the supports of the spinors $\psi_{\mu}, \mu=$ $1, \ldots, N$ all lie in the ball of radius $b N^{1 / 3}$ centered at $\lambda N^{1 / 3} \boldsymbol{e}$ where $b=1 / 2$ if $N \geq 1.2 \cdot 10^{7}$, $b=3 / 5$ if $N \geq 5 \cdot 10^{3}$ and $b=\sqrt{3}$ if $N \geq 1$ (the ball of radius $\sqrt{3} n^{1 / 3}$ contains never less than $n$ lattice cubes). This replaces equation (4) and implies, together with equations (7) and (8), that

$$
\left|\widehat{\boldsymbol{J}}_{\mu}(\boldsymbol{p})-\widehat{\boldsymbol{J}}_{0}(\boldsymbol{p})\right| \leq \frac{6 b}{\lambda-b}\left|\widehat{\boldsymbol{J}}_{0}(\boldsymbol{p})\right|, \quad \lambda>b .
$$

Using this and $\left|\widehat{\boldsymbol{J}}_{0}(\boldsymbol{p})\right|=1 / 2(2 \pi)^{-3 / 2}(1-p)^{2}(2+p)$ one finds

$$
\begin{aligned}
\int \frac{\boldsymbol{J}_{T}(\boldsymbol{x}) \boldsymbol{J}_{T}(\boldsymbol{y})}{|\boldsymbol{x}-\boldsymbol{y}|} d \boldsymbol{x} d \boldsymbol{y} & =\sum_{\mu, \nu=1}^{N} \int d \boldsymbol{p} \frac{4 \pi}{\boldsymbol{p}^{2}} \widehat{\boldsymbol{J}}_{\mu}^{*}(\boldsymbol{p}) T \widehat{\boldsymbol{J}}_{\nu}(\boldsymbol{p}) \\
& \geq N^{2}\left[\int d \boldsymbol{p} \frac{4 \pi}{p^{2}} \widehat{\boldsymbol{J}}_{0}^{*}(\boldsymbol{p}) T \widehat{\boldsymbol{J}}_{0}(\boldsymbol{p})-\frac{12 b}{\lambda-b} \int d \boldsymbol{p} \frac{4 \pi}{p^{2}}\left|\widehat{\boldsymbol{J}}_{0}(\boldsymbol{p})\right|^{2}\right] \\
& =N^{2}\left[1-\frac{18 b}{\lambda-b}\right] \frac{11}{35 \pi},
\end{aligned}
$$

where $T$ is the $3 \times 3$ matrix with the components $T_{i j}=\delta_{i j}-p_{i} p_{j} / p^{2}$. This replaces (17). We proceed to bound the self-energy and exchange terms. Inequality (19) becomes

$$
\left|\widehat{\boldsymbol{J}}_{\mu \nu}(\boldsymbol{p})\right|^{2} \leq 3(2 \pi)^{-3} \chi\left(\left|\boldsymbol{p}+\boldsymbol{n}_{\mu}-\boldsymbol{n}_{\nu}\right| \leq 1\right),
$$

because the support of $u$ now has diameter 1 not $\sqrt{3}$. Since the $N$ balls $B\left(\boldsymbol{n}_{\nu}, 1\right)$ cover a given point at most 8 times (recall that now $\boldsymbol{n}_{2 \nu-1}=\boldsymbol{n}_{2 \nu}$ ) inequality (A.2) leads to the bound

$$
\frac{1}{2} \sum_{\mu, \nu}\left\langle\psi_{\mu} \otimes \psi_{\nu}, B(x-y) \psi_{\nu} \otimes \psi_{\mu}\right\rangle \leq \frac{48}{\pi} b N^{4 / 3}
$$


improving (20). The kinetic energy is bounded by $(\lambda+b) N^{4 / 3}$ and $\left\langle\psi, V_{c} \psi\right\rangle \leq 0$ since $\sum Z_{\kappa}=N$ and $\sum Z_{\kappa}^{2} \geq 1$. In conjunction with (A.1) and (A.3) this gives

$$
\mathcal{E}_{N}(\psi) \leq N^{4 / 3}\left[\lambda+b+\frac{48}{\pi} b \alpha-\alpha N^{2 / 3} \frac{11}{70 \pi}\left(1-\frac{18 b}{\lambda-b}\right)\right] .
$$

For $b=1 / 2, \alpha^{-1}=137$ and the optimal $\lambda$ this is negative for $N \geq 3.4 \cdot 10^{7}$. For $b=3 / 5$ and $\alpha>0$ arbitrary this is negative for $N \geq C \max \left(\alpha^{-3 / 2}, 1\right)$ with $C=43859$ where $\lambda$ was chosen to minimize $C$. This explains the numbers in Theorem 3 .

Now drop the term $(48 / \pi) b \alpha$ in equation (A.4) which was due to the exchange- and selfenergy terms. By equation (11) what we are left with is a upper bound for $\inf _{\psi, \boldsymbol{A} \in \mathcal{A}} \mathcal{E}_{N}(\psi, \boldsymbol{A})$. It is negative for $b=\sqrt{3}$, the optimal $\lambda$ and $\alpha^{3 / 2} N \geq 134^{\prime} 863$, or for $b=1 / 2$ the optimal $\lambda$, $\alpha^{-1}=137$, and $N \geq 3.4 \cdot 10^{7}$. This explains the numbers in the remark after Corollary 2 .

\section{References}

[1] Freeman J. Dyson and Andrew Lenard. Stability of matter I. J. Math. Phys., 8:423-434, 1967.

[2] Freeman J. Dyson and Andrew Lenard. Stability of matter II. J. Math. Phys., 9:698$711,1967$.

[3] Elliott H. Lieb, Heinz Siedentop, and Jan Philip Solovej. Stability and instability of relativistic electrons in classical electromagnetic fields. to appear in J. Stat. Phys., 1997.

[4] J. Fröhlich, E. H. Lieb, and M. Loss. Stability of Coulomb systems with magnetic fields I. the one-electron atom. Commun. Math. Phys., (104):251-270, 1986.

[5] Elliott H. Lieb and Michael Loss. Stability of Coulomb systems with magnetic fields. II: The many-electron atom and the one-electron molecule. Commun. Math. Phys., 104:271-282, 1986.

[6] Elliott H. Lieb and Horng-Tzer Yau. The stability and instability of relativistic matter. Commun. Math. Phys., 118:177-213, 1988.

[7] Michael Loss. The stability of matter interacting with fields. Notices Amer. Math. Soc., 44(10):1288-1293, 1997.

[8] J. Sucher. Foundations of the relativistic theory of many-electron atoms. Phys. Rev. A, 22(2):348-362, August 1980.

[9] J. Sucher. Relativistic many-electron Hamiltonians. Phys. Scripta, 36:271-281, 1987.

[10] Y. Ishikawa and K. Koc. Relativistic many-body perturbation theory based on the nopair Dirac-Coulomb-Breit Hamiltonian: Relativistic correlation energies for the noblegas sequence through $\mathrm{Rn}(Z=86)$, the group-II $B$ atoms through $\mathrm{Hg}$, and the ions of Ne isoelectronic sequence. Phys. Rev. A, 50(6):4733-4742, December 1994.

[11] Yasuyuki Ishikawa and Konrad Koc. Relativistic many-body perturbation calculations for open-shell systems. Phys. Rev. A, 56(2):1295-1304, 1997.

[12] P. Pyykkö. Relativistic effects in structural chemistry. Chem. Rev., (88):563-594, 1988.

[13] William Desmond Evans, Peter Perry, and Heinz Siedentop. The spectrum of relativistic one-electron atoms according to Bethe and Salpeter. Commun. Math. Phys., 178(3):733-746, July 1996. 
[14] C. Tix. Strict positivity of a relativistic Hamiltonian due to Brown and Ravenhall. to appear in Bull. London Math. Soc., 1998.

[15] C. Tix. Lower bound for the ground state energy of the no-pair hamiltonian. Physics Letters B, (405):293-296, 1997.

[16] A. A. Balinsky and W. E. Evans. Stability of one-electron molecules in the BrownRavenhall model. preprint, 1998.

[17] A. A. Balinsky and W. E. Evans. On the virial theorem for the relativistic operator of Brown and Ravenhall, and the absence of embedded eigenvalues. Lett. Math. Phys., 44:233-248, 1998.

[18] M. Griesemer and H. Siedentop. A minimax principle for eigenvalue in spectral gaps. to appear in Jour. London Math. Soc., 1997.

[19] G. Nenciu and G. Scharf. On regular external fields in quantum electrodynamics. Helv. Phys. Acta, 51(3):412-424, 1978.

[20] W. Appel and A. Alastuey. Relativistic corrections for a classical one-component plasma. Physica A, 252:238-268, 1998.

[21] M. Kiessling. private cummunication, 1998.

[22] L.D. Landau and E.M. Lifshitz. The classical theory of fields. Pergamon Press, 1971.

[23] Hans A. Bethe and Edwin E. Salpeter. Quantum mechanics of one- and two-electron atoms. In S. Flügge, editor, Handbuch der Physik, XXXV, pages 88-436. Springer, Berlin, 1 edition, 1957.

[24] Volker Bach, Jürg Fröhlich, and Israel Michael Sigal. Quantum electrodynamics of confined nonrelativistic particles. Adv. Math., 137(2):299-395, 1998. 